\title{
Erythematous Papules Involving the Eyebrows in a Patient with a History of Rosacea and Hair Loss
}

\author{
Agata Kłosowicz $^{\mathrm{a}}$ Curtis Thompson $^{\mathrm{b}}$ Antonella Tosti ${ }^{\mathrm{c}}$ \\ ${ }^{a}$ Department of Dermatology, University Hospital in Kraków, Kraków, Poland; ' ${ }^{\text {CTA }}$ Lab, Portland, OR, USA; \\ ${ }^{C}$ Dr. Phillip Frost Department of Dermatology and Cutaneous Surgery, University of Miami Miller School of \\ Medicine, Miami, FL, USA
}

\section{Question}

A 47-year-old woman presented with a 2-year history of hair loss and rosacea. She had been treated with topical metronidazole without improvement. Clinical examination revealed very sparse eyebrows and grouped small erythematous papules on the top of her eyebrows bilaterally (Fig. 1a). She had prominent telangiectasias of the cheeks and temporal hairline recession. A dermoscopyguided 2-mm biopsy (Fig. 1b) from an erythematous papule showed a dilated follicular infundibulum, and some surrounding lymphocytes with focal vacuolization of the follicular epithelial basalis without significant perifollicular fibrosis (Fig. 2a). Some demodex material was identified within the follicular ostia. Large sebaceous lobules were present in the central, subjacent dermis with associated adnexal glands (Fig. 2b).

What is your diagnosis?

(1) Papulopustular rosacea; (2) facial papules of frontal fibrosing alopecia (FFA); (3) periorificial dermatitis; or (4) ulerythema ophryogenes.
Agata Kłosowicz, MD

Department of Dermatology, University Hospital in Kraków Skawinska 8

PL-31-066 Kraków (Poland)

klosowiczagata@gmail.com 

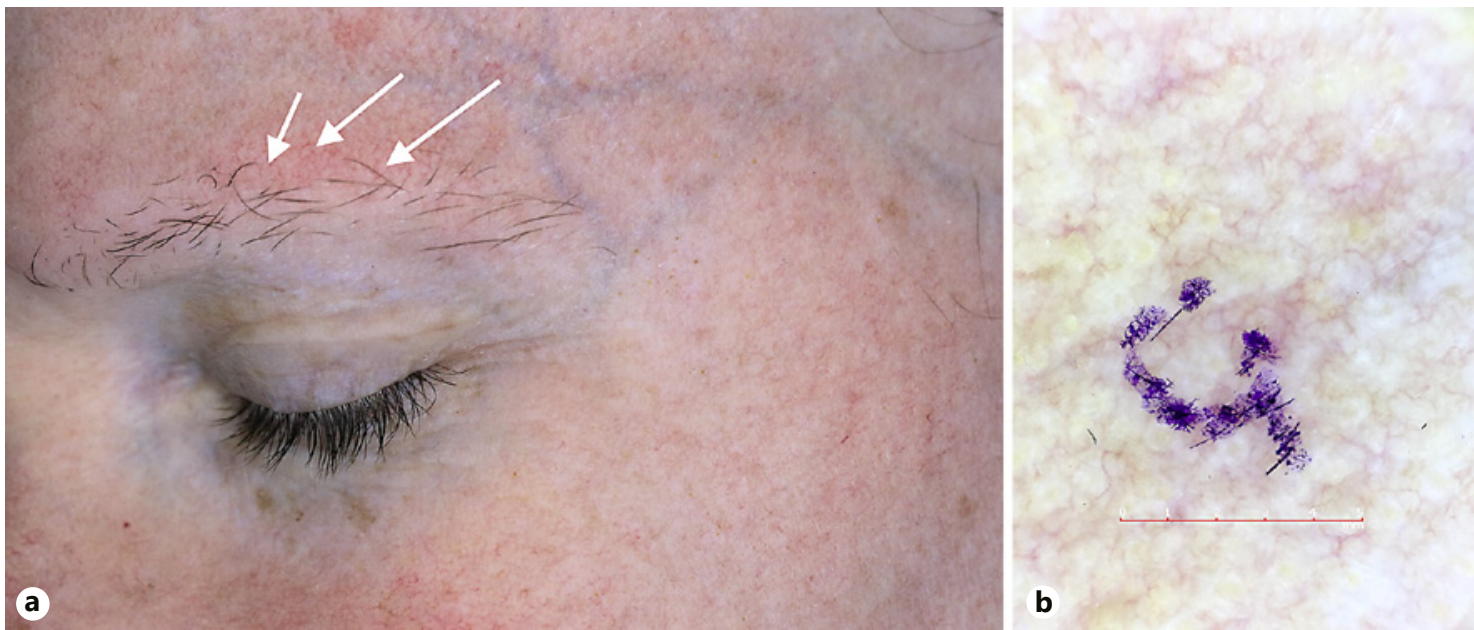

Fig. 1. a Diffuse alopecia of the eyebrow and grouped 1-mm papules with a prominent erythematous border. b Dermoscopic image of a facial papule with erythematous border.
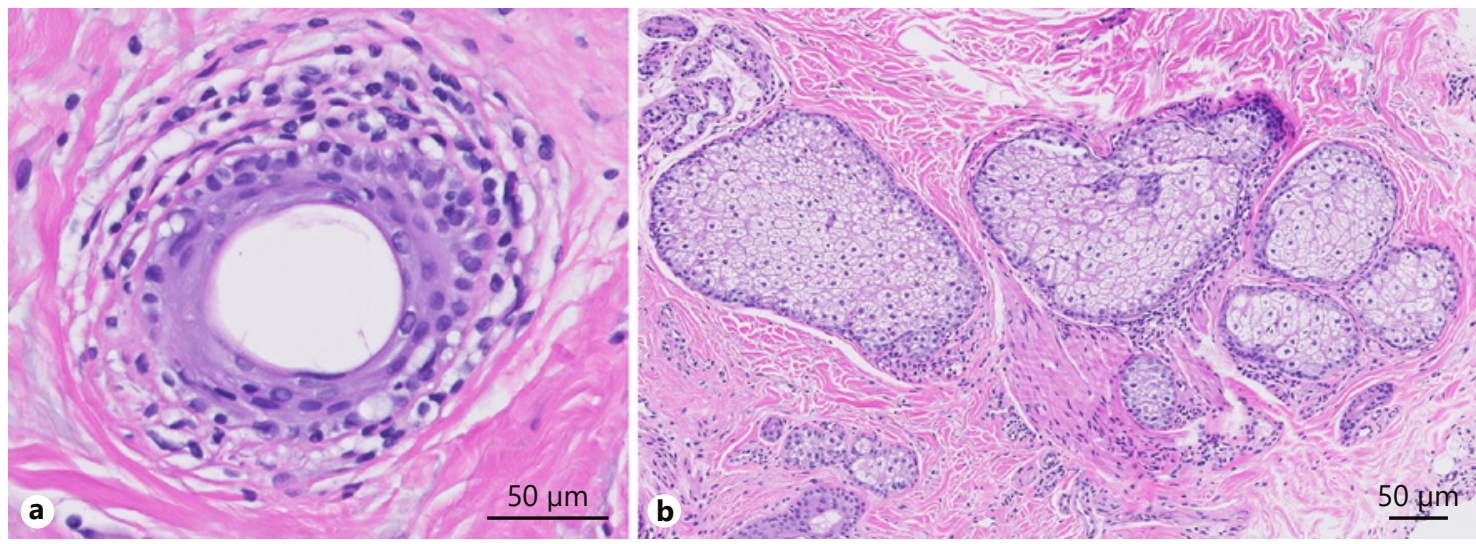

Fig. 2. a Vacuolization of the follicular epithelial basalis with subjacent lymphocytes in the superficial reticular dermis. b Large subjacent sebaceous lobules in the mid-reticular dermis. 


\section{Answer}

The answer is: FFA. Histopathologic examination revealed folliculocentric interface change with lymphocytes, thereby fulfilling criteria for a diagnosis of FFA (Fig. 2a). Sebaceous hyperplasia is a usual finding in facial papules of FFA (Fig. 2b).

FFA, first described by Kossard in 1994, is considered a clinically distinct variant of lichen planopilaris that mostly affects postmenopausal women [1]. The incidence rate of FFA has markedly increased in the past decade. FFA presents as a progressive band-like alopecia of the frontal hairline often associated with the loss of the eyebrows [2]. Facial lesions are commonly observed with variable incidences ranging from 22 to $41 \%$ [3, 4]. Facial papules are the most common skin findings reported in FFA patients, especially in those with dark phototypes [4].

In the largest series of FFA patients to date, VañóGalván et al. [2] reported the presence of facial papules in $14 \%$ of cases. According to the authors, facial papules are associated with higher disease activity and more severe forms of FFA [2]. López-Pestaña et al. [3] observed the presence of noninflammatory facial papules in $20 \%$ of FFA patients. They suggested that the recognition of these discrete and asymptomatic lesions is easier in premenopausal women due to the fact that solar elastosis and wrinkles can hamper the diagnosis in aged patients [3]. Noninflammatory dome-shaped papules on the temples and cheeks were also the most common facial lesions found in an ethnically diverse FFA series, particularly prevalent in the Hispanic/Latin population (41\%) [4].

In 2011, Donati et al. [5] described lichenoid perifollicular inflammation and fibrosis in skin biopsies of facial papules from 4 FFA patients. They hypothesized that facial papules result from lichenoid inflammation of facial vellus hair follicles. This finding was not confirmed by two more recent studies that found that facial papules correspond to hypertrophic sebaceous glands without inflammatory changes and reported the efficacy of isotretinoin in their treatment. In our case, the patient was prescribed low-dose isotretinoin $(10 \mathrm{mg} /$ day). The histopathological features were analyzed in skin biopsies of facial papules from 3 [6] and 10 [7] FFA patients. Pirmez et al. [8] hypothesized that the formation of facial papules is due to an abnormal elastic framework that remodels the shape of sebaceous lobules and ducts.

FFA has been described as occurring in association with rosacea. In a descriptive cross-sectional study, rosacea was the most frequently associated cutaneous condi- tion in 103 patients with FFA, with a prevalence of $34 \%$. The most common subtype of the disease in FFA patients was erythematotelangiectatic rosacea, followed by papulopustular rosacea. The authors suggest that FFA and rosacea might share a common pathway of the pathogenesis of pilosebaceous unit diseases [9]. Histologically, enlarged sebaceous glands often present in patients with rhinophyma and are not characteristic of papulopustular rosacea [10].

Our case illustrates that facial papules of FFA might be erythematous in patients with light phototypes, and that a distinction between papulopustular rosacea and FFA may be difficult if not impossible without histopathology.

\section{Statement of Ethics}

The authors state that the patient has given her written consent to publish photos and details of the case.

\section{Disclosure Statement}

The authors have no conflicts of interest to declare.

\section{Funding Sources}

No funding was received.

\section{Author Contributions}

Article conception and design: A. Tosti, A. Kłosowicz, C. Thompson. Acquisition of data: A. Tosti, C. Thompson, A. Kłosowicz. Analysis and interpretation: A. Kłosowicz, A. Tosti, C. Thompson. Preparation of the outline of the manuscript: A. Kłosowicz, A. Tosti, C. Thompson. Literature review: A. Kłosowicz, A. Tosti. Critical review and supervision: A. Tosti, C. Thompson.

\section{Keywords}

Facial papules · Frontal fibrosing alopecia $\cdot$ Scarring alopecia $\cdot$ Clinicopathological correlation 


\section{References}

1 Kossard S, Lee MS, Wilkinson B. Postmenopausal frontal fibrosing alopecia: a frontal variant of lichen planopilaris. J Am Acad Dermatol. 1997 Jan;36(1):59-66.

2 Vañó-Galván S, Molina-Ruiz AM, SerranoFalcón C, Arias-Santiago S, Rodrigues-Barata AR, Garnacho-Saucedo G, et al. Frontal fibrosing alopecia: a multicenter review of 355 patients. J Am Acad Dermatol. 2014 Apr; 70(4):670-8.

3 López-Pestaña A, Tuneu A, Lobo C, Ormaechea N, Zubizarreta J, Vildosola S, et al. Facial lesions in frontal fibrosing alopecia (FFA): clinicopathological features in a series of 12 cases. J Am Acad Dermatol. 2015 Dec;73(6): 987.e1-6.
4 Mervis JS, Borda LJ, Miteva M. Facial and Extrafacial Lesions in an Ethnically Diverse Series of 91 Patients with Frontal Fibrosing Alopecia Followed at a Single Center. Dermatology. 2019;235(2):112-9.

5 Donati A, Molina L, Doche I, Valente NS, Romiti R. Facial papules in frontal fibrosing alopecia: evidence of vellus follicle involvement. Arch Dermatol. 2011 Dec;147(12): 1424-7.

6 Pirmez R, Duque-Estrada B, Barreto T, Quintella DC, Cuzzi T. Successful Treatment of Facial Papules in Frontal Fibrosing Alopecia with Oral Isotretinoin. Skin Appendage Disord. 2017 May;3(2):111-3.

7 Pedrosa AF, Duarte AF, Haneke E, Correia O. Yellow facial papules associated with frontal fibrosing alopecia: A distinct histologic pattern and response to isotretinoin. J Am Acad Dermatol. 2017 Oct;77(4):764-6.
8 Pirmez R, Barreto T, Duque-Estrada B, Quintella DC, Cuzzi T. Facial papules in frontal fibrosing alopecia: beyond vellus hair follicle involvement. Skin Appendage Disord. 2018 Aug;4(3):145-9.

9 Pindado-Ortega C, Saceda-Corralo D Buendía-Castaño D, Fernández-González P, Moreno-Arrones ÓM, Fonda-Pascual P, et al. Frontal fibrosing alopecia and cutaneous comorbidities: A potential relationship with rosacea. J Am Acad Dermatol. 2018 Mar;78(3): 596-597.e1.

10 Tope WD, Sangueza OP. Rhinophyma's fibrous variant. Histopathology and immunohistochemistry. Am J Dermatopathol. 1994 Jun;16(3):307-10. 